\title{
Direct imaging of skyrmion in plan-view of a polycrystalline FeGe thin film
}

Binbin wang ${ }^{1}$, Núria Bagués ${ }^{2}$, Roland Kawakami ${ }^{3}$ and David McComb ${ }^{2}$

${ }^{1}$ The Ohio State University, COLUMBUS, Ohio, United States, ${ }^{2}$ Center for Electron Microscopy and Analysis, The Ohio State University, Columbus, OH, USA, COLUMBUS, Ohio, United States, ${ }^{3}$ The Ohio State University, United States

Real space imaging of magnetic textures such as skyrmions is important for characterization and development of magnetic and spintronic devices. In the transmission electron microscopy (TEM) techniques to image magnetic textures are generally called Lorentz modes, and include Fresnel imaging, Foucault imaging, and electron holography [1]. All these techniques have been successfully used to observe nanometric topological magnetic configurations in variety material systems, such as vortices, bubbles and skyrmions [2].

The desire to design and build skyrmion-based devices is driving the need to characterize magnetic textures in thin film of functional materials. Utilizing current LTEM methods in thin layers with complex microstructures where relatively weak magnetic contrast can be overwhelmed by diffraction contrast is challenging. Consequently, methods to enhance and disentangle the weak magnetic contrast in plan-view thin films from a complex background contrast are increasingly importance. Generally, this can be realized in the post-processing of image data sets. For example, in Lorentz TEM images, methods for background subtraction method and Fourier filtering have been demonstrated to be effective in single crystals and, in some cases, thin films [3,4]. For scanning TEM (STEM) data, methods, such as frequency filtering by selection of different scattering angle ranges in the transmitted disk [5-7] and specimen-tilt series averaging differential phase contrast (DPC) STEM [8] have been explored.

In this work, we have employed both Lorentz TEM and Lorentz STEM to study the magnetic texture in two FeGe systems. FeGe is a chiral magnet with B20 structure and a Curie temperature of $\sim 280 \mathrm{~K}$ [9]. In Fig.1, we show two examples of resolved skyrmion phase mapping on single crystal FeGe using both Lorentz TEM and Lorentz STEM. The magnetic contrast in this single crystal is very clear and enables images showing the magnetic field strength to be reconstructed via the transport of intensity equation in Lorentz TEM or center of mass (CoM)-DPC in Lorentz STEM. Our second example is a polycrystalline FeGe thin film $(35 \mathrm{~nm})$ grown on Si substrate. Direct imaging of the magnetic texture in this plan-view sample proved to be much more challenging due to the complex "background" contrast associated with the defects, as well artifacts such as bending and polishing damage, as shown in Fig.2. To disentangle magnetic contrast from the background contrast, we explored three post-processing methods: 1) background subtraction in defocused Lorentz-TEM images; 2) frequency filtered CoM-DPC reconstructed from Lorentz-STEM datasets; 3) registration of 4D-STEM datasets acquired at different tilting angles. Using these methods, we successfully achieved real space imaging of both helical and skyrmion phases in this plan-view specimen, an observation that contrasts with a previous report [5]. The capacity of understanding the magnetic behavior at nanoscale from plan-view thin films is a fundamental step for dynamic imaging of motion in skyrmion-based spintronic devices.

The authors acknowledge funding from Defense Advanced Research Projects Agency (DARPA) under Grant No. D18AP00008 and the Center for Emergent Materials at The Ohio State University, an NSF MRSEC (DMR-2011876). 

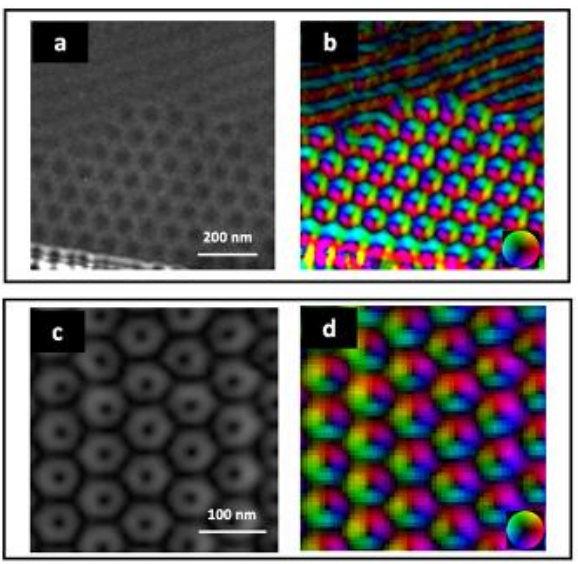

Figure 1. Real space imaging of magnetic textures in single crystal FeGe at $240 \mathrm{~K}$ : (a) over-focused LTEM image of a coexist of skyrmion and helical spin texture; (b) magnetic reconstruction maps obtained from defocused LTEM images in (a) (respective under-focus image not shown here); (c) Reconstructed magnetic field strength of skyrmion spin textures and corresponding polar figure in (d) from CoM-DPC of 4dSTEM dataset. The color wheel indicates the field direction.

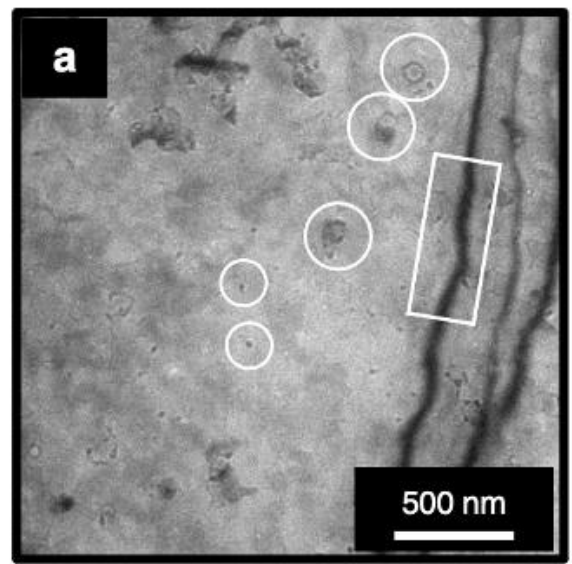

Defocus $=0 \mu m$

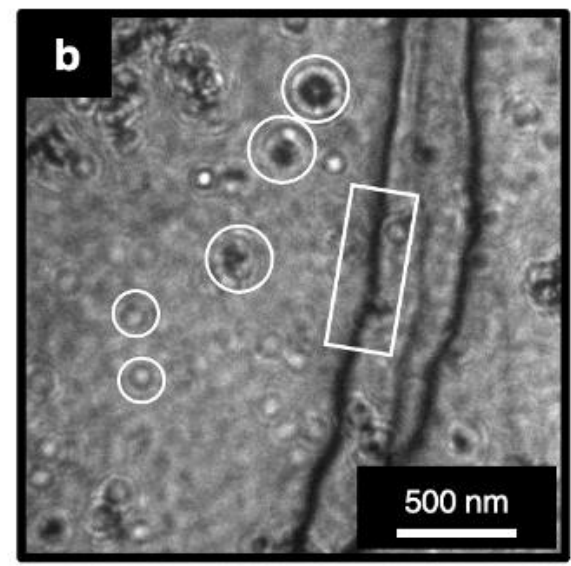

Defocus $=400 \mu \mathrm{m}$

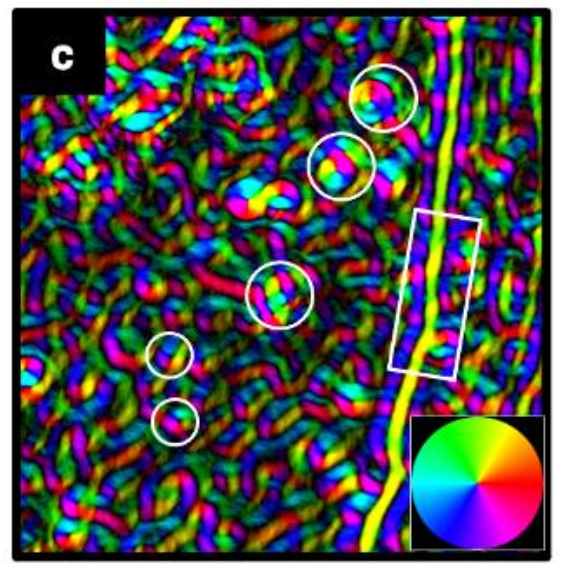

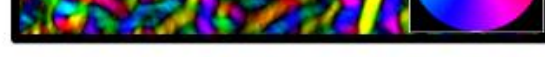

Figure 2. Plan-view LTEM series images form the same area of a polycrystalline FeGe thin film acquired at $240 \mathrm{~K}, 80 \mathrm{mT}$ : (a) in-focus, (b) $400 \mu \mathrm{m}$ over-focused. Respective under-focus images not shown here. (c) $80 \mathrm{mT}$ magnetic field reconstruction maps obtained from defocused LTEM. The color wheel indicates the field direction. The white circle in the images highlighted same residual particles as reference markers while the white rectangle highlighted a bend contour.

\section{References}

[1] Jin Tang, et al.,Chinese Phys. B 28 (2019), p. 087503

[2] Li-cong Peng, et al. Chinese Phys. B 27 (2018), p. 066802

[3] N. Bagues, et al., Microsc. Microanal 26 (2020), p. 1700.

[4] M. Hirschberger, et al., Nat. Commun. 10 (2019), p.1.

[5] M. Krajnak, , Ultramicroscopy 165 (2016), p.42.

[6] K. X. Nguyen et al., arXiv preprint arXiv:200106900, (2020).

[7] B. Wang, et al., Microsc. Microanal. 26 (2020), p. 1902.

[8] Y. O. Murakami, Microscopy 69 (2020), p. 312.

[9] X. Z. Yu, et al., Nature 465 (2010), p. 901. 